Rapid Reviews COVID-19

\title{
Reviews of "Safety and efficacy of the ChAdOx1 nCoV-19 (AZD1222) Covid- 19 vaccine against the B.1.351 variant in South Africa"
}

\author{
Alfonso J. Rodriguez-Morales ${ }^{\mathbf{1}}$, Julian Ruiz-Saenz ${ }^{2}$, Roy Soiza ${ }^{\mathbf{3}}$ \\ ${ }^{1}$ Faculty and Senior Researcher, Fundacion Universitaria Autonoma de las Americas, Faculty of \\ Medicine, Colombia, \\ 2Universidad Cooperativa de Colombia, Medicina Veterinaria, Colombia, \\ ${ }^{3}$ Hon Clinical Reader, University of Aberdeen, Institute of Applied Health Sciences, UK
}

Published on: Apr 20, 2021

License: Creative Commons Attribution 4.0 International License (CC-BY 4.0). 
To read the original manuscript, click the link above.

Summary of Reviews: This preprint suggests the Astra-Zeneca vaccine had little efficacy against non-hospitalized mild to moderate Covid-19 due to B.1.351. Reviewers found it was potentially informative, suggesting the need for more studies to assess vaccine effectiveness against the B.1.351 variant.

\title{
Reviewer 1 (Alfonso J. Rodriguez-Morales) | |
}

Reviewer 2 (Julian Ruiz-Saenz) |

\section{Reviewer 3 (Roy Soiza) | |}

\author{
प्रमप्र = Misleading \\ प्र००० = Not Informative

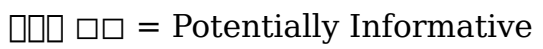 \\ प्रीप् $=$ Reliable \\ प्राप्र = Strong
}

RR:C19 Strength of Evidence Scale Key.

To read the reviews, click the links below. 